\title{
A perfect tool for comprehensive evaluation of myocardial perfusion and function: Stress PET imaging
}

\author{
Guang-Uei Hung, MD, FANMB, ${ }^{a}$ Weihua Zhou, PhD, ${ }^{\mathrm{b}}$ and Ji Chen, $\mathrm{PhD}^{\mathrm{c}}$ \\ a Department of Nuclear Medicine, Chang Bing Show Chwan Memorial Hospital, Changhua, \\ Taiwan \\ b School of Computing, University of Southern Mississippi, Long Beach, MS \\ c Department of Radiology and Imaging Sciences, Emory University School of Medicine, Atlanta, \\ GA
}

Received Dec 12, 2018; accepted Dec 13, 2018

doi: $10.1007 / \mathrm{s} 12350-018-01580-0$

\section{See related article, pp. 2234-2242}

Pharmacologic stress cardiac positron emission tomography (PET) is a highly advanced technique for myocardial perfusion imaging (MPI). In comparison with traditional single-photon emission computed tomography (SPECT), MPI with PET provides faster acquisition, better quality, less attenuation artifact, lower radiation burden, and more accurate quantitation of myocardial blood flow (MBF) and perfusion reserve (MPR). With all the advantages, MPI with PET brings higher diagnostic accuracy and more accurate risk stratification and decision-making to patients with known or suspected coronary artery disease (CAD). ${ }^{1-3}$ In addition, applying ECG-gating technique further enables MPI with PET to simultaneously assess left ventricular (LV) functions and synchrony not only at resting but also at peak-stress status. ${ }^{4,5}$ All of these myocardial substrates produce additional values in diagnosis and prognosis ${ }^{6,7}$ and also provide wonderful opportunities for researches on the pathophysiology mechanisms in patients with CAD and heart failure.

In this issue of journal of nuclear cardiology, Juarez-Orozco et al used adenosine-stress N-13 ammonia PET to retrospectively study the relationship

Reprint requests: Guang-Uei Hung, MD, FANMB, Department of Nuclear Medicine, Chang Bing Show Chwan Memorial Hospital, No. 6, Lukong Road, Lukang Town, Changhua 505, Taiwan; 106143@gmail.com

J Nucl Cardiol 2020;27:2243-6.

$1071-3581 / \$ 34.00$

Copyright (C) 2019 American Society of Nuclear Cardiology. between traditional perfusion estimate with summed rest score (SRS, a surrogate of myocardial scar) and quantitative perfusion estimates with stress MBF (sMBF), rest MBF (rMBF), MPR, and peak-stress ventricular synchrony expressed as bandwidth (BW), standard deviation (SD), and entropy (E) in chronic heart failure patients referred for MPI with PET due to suspected myocardial ischemia. ${ }^{8}$ The authors found an inverse relationship between perfusion estimates and ventricular synchrony. However, quantitative estimates with sMBF, $\mathrm{rMBF}$, and MPR were inferior to SRS for predicting ventricular mechanical synchrony in these patients. The authors further proposed that characterizing the fixed perfusion defects with SRS might be a more convenient approach for treatment in order to improve ventricular mechanical dyssynchrony. Interestingly, the same group had a similar study in which the enrolled patients were also referred for N-13 ammonia PET due to suspected myocardial ischemia but not limited to chronic heart failure ${ }^{9}$ however, sMBF is better than MPR, SSS, and SRS in predicting peak-stress ventricular synchrony independently from other relevant cardiovascular risk factors and clinical covariates. The different results between the authors' two studies might be caused by the more complicated mechanism of ventricular synchrony in patients with chronic heart failure and the further study is needed to answer this question.

The relationship between myocardial perfusion status and LV mechanical synchrony had been well described in previous studies. Our previous study with SPECT showed that stress-induced myocardial ischemia caused dyssynchronous contraction in the ischemic region, deteriorating $\mathrm{LV}$ mechanical synchrony. ${ }^{10}$ Moreover, our study showed that LV dyssynchrony at stress was more significantly reduced than that at rest in 

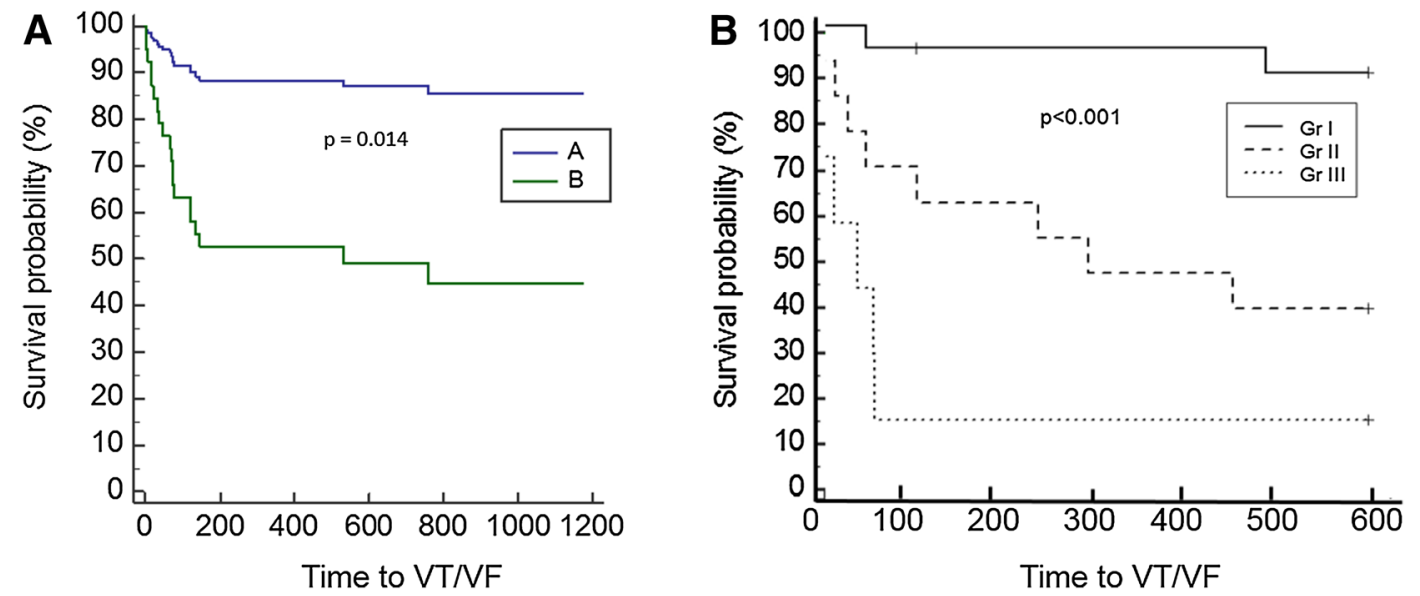

Figure 1. A Kaplan-Meier survival curves for time from MPI to ventricular arrhythmia for the patients with $\mathrm{EF}>29 \%$, scar $>23 \%$, and phase $\mathrm{SD}>50^{\circ}$ (group A) and the others (group B). B Kaplan-Meier survival curves for time from MPI to ventricular arrhythmia for patients with 2 risk factors (group I), 3 to 4 risk factors (group II), and 5 risk factors (group III). Reproduced with permission from Hou et al. Ann Nucl Med 2015;29:772-8 [Figure 3] ${ }^{11}$ and Chiang et al. J Nucl Cardiol 2017;24:1282-8 [Figure 2]. ${ }^{17}$

the normal and infarcted myocardium. For patients with chronic heart failure, however, the current study found myocardial scar (the extent and severity of fixed perfusion defects as SRS) was even more important in the pathophysiologic mechanism of ventricular synchrony. In our previous SPECT studies, we had similar observations that significant correlation was noted between myocardial scar (expressed as area of resting myocardial perfusion defect) and phase SD in heart failure patients with cardiac resynchronization therapy $(\mathrm{CRT}){ }^{11}$ In addition, we further demonstrated that myocardial scar interfered with the normal propagation of mechanical activation, resulting in more heterogeneous activation sequences and thus contributing to the development of ventricular arrhythmia. ${ }^{12}$

Since the invention of the phase analysis technique from ECG-gated MPI with SPECT by Chen et al in $2005,{ }^{13}$ it has led this imaging modality to discover a "New World"' in nuclear cardiology. LV mechanical dyssynchrony by phase analysis has been found potentially useful in selecting optimal candidates for CRT, guiding LV lead implantation of CRT at the latest activation site, detecting stress-induced worsening of $\mathrm{LV}$ dyssynchrony as a marker of myocardial stunning, and differentiating ischemic or non-ischemic cardiomyopathy, risk stratification, and prognostication in heart failure patients. ${ }^{14}$ For patients with chronic heart failure, one of the major challenges is to predict those who are at the highest risk for developing ventricular arrhythmia and sudden cardiac death and selecting the appropriate candidates for receiving implantable cardioverter defibrillators. It is encouraging that phase analysis has also been found useful in previous studies which showed that the severity of LV dyssynchrony as assessed by phase analysis was related to appropriate ICD shocks and SCD events. $^{15,16}$ Our group further demonstrated that myocardial scar burden (area with activity less than 50\% of maximal myocardial uptake), LVEF, and LV dyssynchrony as assessed by gated SPECT MPI were significantly correlated to the development of ventricular arrhythmia (ventricular tachycardia or ventricular fibrillation) in patients with heart failure who had received CRT. ${ }^{11}$ As shown in Figure 1A, those (group A) with better ventricular systolic function (LVEF $>29 \%$ ), smaller infarcted myocardium (scar burden $<23 \%$ ), and less LV dyssynchrony (phase $\mathrm{SD}<50^{\circ}$ ) had significantly better survival than the others (group B) for the development of ventricular arrhythmia. The latter (group B) had a hazard ration of 5.16 (compared to group A). Incorporating findings of SPECT, ECG, and echocardiography, our group developed a risk factor-based model to predict those patients at the highest risk of ventricular arrhythmia. ${ }^{17}$ Five independent predictors of ventricular arrhythmia (risk factors) include LVEF after CRT $\leq 30 \%$, phase $\mathrm{SD} \geq 45.6^{\circ}, \Delta \mathrm{iQRSd} \leq 7 \mathrm{~ms}$, iQRSd after $\mathrm{CRT} \geq 121 \mathrm{~ms}$, and $\triangle \mathrm{LVEF} \leq 7 \%$. On KaplanMeier survival analysis for time to ventricular arrhythmia (Figure 1B), an increasing number of these risk factors were associated with significantly more events (Group I: 2 risk factors; Group II: 3 to 4 risk factors; Group III: 5 risk factors; $p<0.001)$. The annualized rate of arrhythmic events increased from approximately $6 \%$ for 2 risk factors, $35 \%$ for 3 to 4 risk factors to $53 \%$ for 5 risk factors. Figure 2 illustrates an example image of the studied 


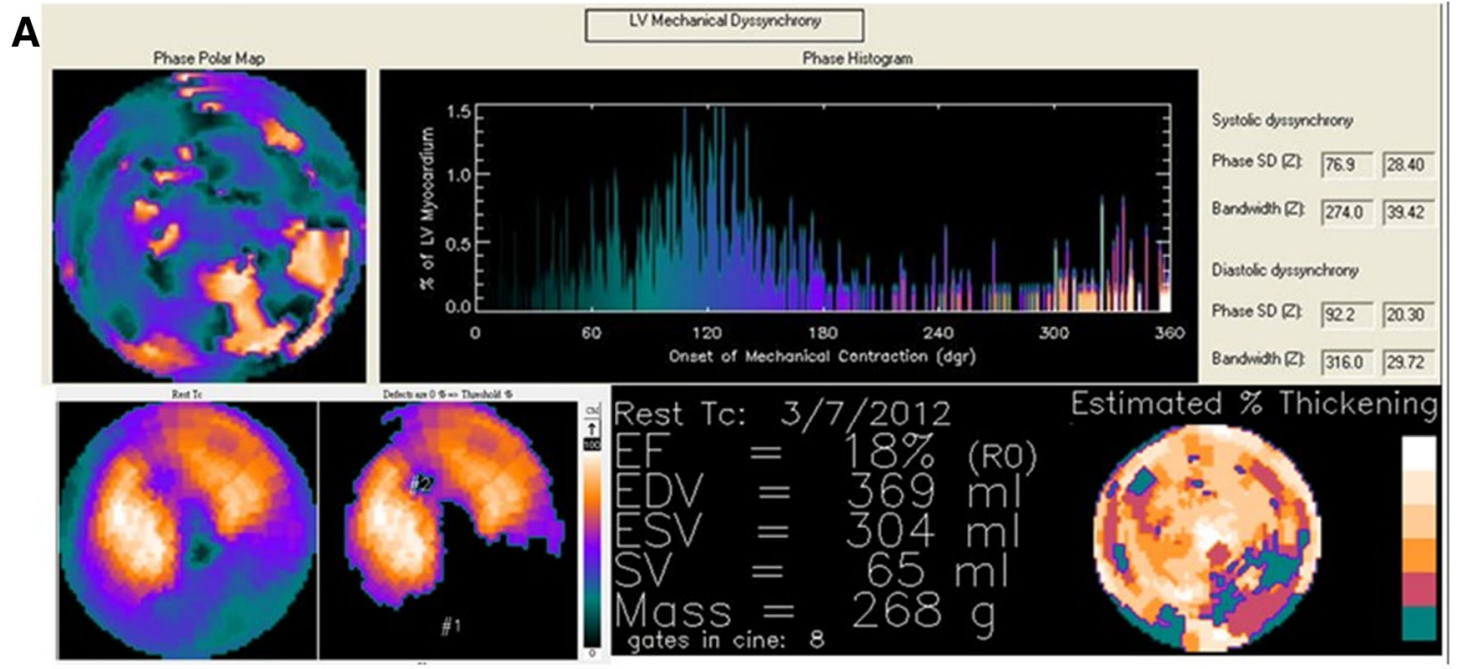

B

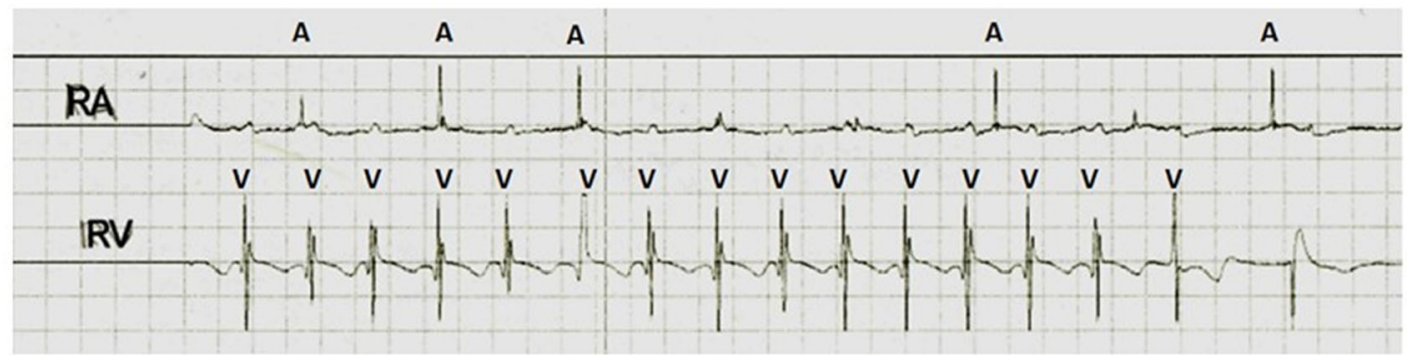

Figure 2. A 42-year-old male patient received resting MPI with ECG-gated SPECT 10 months after CRT implantation. The results show a large scar area of $46 \%$, a low LVEF of $18 \%$, and large phase SD of $92^{\circ}$ (A). He was found to develop ventricular tachycardia (VT) during follow-up and died of sudden cardiac death later. B The patient's ECG recorded by CRT device shows a picture of VA dissociation. The atrial rate (A rate) on RA lead, ranged from 800 to $2400 \mathrm{~ms} / \mathrm{cycle}$, is much slower than the ventricular rate (V rate), 360 to $390 \mathrm{~ms} /$ cycle. These findings are indicative of an episode of VT. Reproduced with permission from Hou PN, et al. Ann Nucl Med 2015;29:772-8 [Figure 4]. ${ }^{11}$

patients whose SPECT with MPI showed a large perfusion defect (scar burden: $46 \%$ ), very poor $\mathrm{LV}$ systolic function (LVEF: 18\%), and severe LV dyssynchrony (phase SD: $92^{\circ}$ ), and the patient was noted to develop ventricular tachycardia recorded by CRT device during follow-up. With these preliminary data, we believe that a multicenter clinical trial is needed to develop a predictive model using the quantitative parameters of myocardial substrates generated from MPI, especially using the "perfect" tool of PET.

Compared to SPECT imaging mostly using Tc-99m agents, PET has another advantage of imaging cardiac function in the immediate peak-stress condition. Using Rb-92 MPI with PET, Dorbala et al. found that there was inverse relationship between stress-induced change in LVEF and magnitude of ischemia during peak vasodilator stress, and that the stress-induced LVEF worsening highly indicated left main or multivessel CAD and also significantly more cardiac events and all- cause death. ${ }^{18,19}$ On the other hand, Tc-99m SPECT imaging usually starts 30 to 60 minute after stress and often results in recovery of LV stunning with little or no residual detectable change in LV function. Although there are considerable disadvantages including poorer image quality and higher radiation burden to patients, Tl-201 imaging starts 5-10 minute after stress and has more chance to capture stress-induced change than Tc$99 \mathrm{~m}$. Our previous studies using Tl-201 SPECT showed that stress-induced worsening of LVEF or regional wall motion were associated with severe CAD and were independent predictors of major adverse cardiac events. ${ }^{20,21}$ In addition, stress-induced ischemia caused more LV dyssynchronous contraction which provided incremental value in detecting multivessel $\mathrm{CAD}^{22}$ In the current study, the authors studied the relationship between peak-stress LV synchrony and perfusion estimates using N-13 ammonia PET. Using this perfect tool for MPI, we believe it would be even more interesting to 
further explore the pathophysiology mechanism or clinical significance of stress-induced LV dyssynchrony.

It has been more than a decade since phase analysis for MPI with SPECT was invented, and this technique is still expanding its applications in academic research or clinical patient care. In combination with the perfect tool for MPI, stress-rest cardiac PET will provide the most comprehensive information in perfusion, quantitative flow and function than any other nuclear imaging modalities ever. We look forward to the new era of nuclear cardiology with cardiac PET.

\section{Disclosure}

The authors declare that they have no conflict of interest.

\section{References}

1. Bateman TM, Dilsizian V, Beanlands RS, DePuey EG, Heller GV, Wolinsky DA. American Society of Nuclear cardiology and society of nuclear medicine and molecular imaging joint position statement on the clinical indications for myocardial perfusion PET. J Nucl Cardiol 2016;23:1227-31.

2. Taqueti VR, Hachamovitch R, Murthy VL, Naya M, Foster CR, Hainer $\mathbf{J}$, et al. Global coronary flow reserve is associated with adverse cardiovascular events independently of luminal angiographic severity and modifies the effect of early revascularization. Circulation 2015;131:19-27.

3. Gould KL, Johnson NP, Roby A, Nguyen TT, Kirkeeide RL, Haynie M, et al. Regional artery specific thresholds of quantitative myocardial perfusion by PET associated with reduced MI and death after revascularization in stable CAD. J Nucl Med 2018. https://doi.org/10.2967/jnumed.118.211953.

4. AlJaroudi W, Alraies MC, DiFilippo F, Brunken RC, Cerqueira MD, Jaber WA. Effect of stress testing on left ventricular mechanical synchrony by phase analysis of gated positron emission tomography in patients with normal myocardial perfusion. Eur J Nucl Med Mol Imaging 2012;39:665-72.

5. Van Tosh A, Votaw JR, Reichek N, Palestro CJ, Nichols KJ. The relationship between ischemia-induced left ventricular dysfunction, coronary flow reserve, and coronary steal on regadenoson stress-gated $(82) \mathrm{Rb}$ pet myocardial perfusion imaging. J Nucl Cardiol 2013;20:1060-8.

6. AlJaroudi W, Alraies MC, Menon V, Brunken RC, Cerqueira MD, Jaber WA. Predictors and incremental prognostic value of left ventricular mechanical dyssynchrony response during stress-gated positron emission tomography in patients with ischemic cardiomyopathy. J Nucl Cardiol 2012;19:958-69.

7. Goldberg AS, Alraies MC, Cerqueira MD, Jaber WA, Aljaroudi WA. Prognostic value of left ventricular mechanical dyssynchrony by phase analysis in patients with non-ischemic cardiomyopathy with ejection fraction $35-50 \%$ and QRS $<150 \mathrm{~ms}$. J Nucl Cardiol 2014;21:57-66.

8. Juarez-Orozco LE, Monroy-Gonzalez AG, van der Zant FM, Hoogvorst N, Slart RHJA, Knol RJJ. Ventricular synchrony is not significantly determined by absolute myocardial perfusion in patients with chronic heart failure: a ${ }^{13} \mathrm{~N}$-ammonia PET study. J Nucl Cardiol 2018. https://doi.org/10.1007/s12350-018-01507-9.

9. Juárez-Orozco LE, Alexanderson E, Dierckx RA, Boersma HH, Hillege JL, Zeebregts CJ, et al. Stress myocardial blood flow correlates with ventricular function and synchrony better than myocardial perfusion reserve: a Nitrogen-13 ammonia PET study. J Nucl Cardiol 2018;25:797-806.

10. Chen CC, Shen TY, Chang MC, Hung GU, Chen WC, Kao CH, et al. Stress-induced myocardial ischemia is associated with early post-stress left ventricular mechanical dyssynchrony as assessed by phase analysis of $201 \mathrm{Tl}$ gated SPECT myocardial perfusion imaging. Eur J Nucl Med Mol Imaging 2012;39:1904-9.

11. Hou PN, Tsai SC, Lin WY, Cheng CM, Chiang KF, Chang YC, et al. Relationship of quantitative parameters of myocardial perfusion SPECT and ventricular arrhythmia in patients receiving cardiac resynchronization therapy. Ann Nucl Med 2015;29:772-8.

12. Chiang KF, Cheng CM, Tsai SC, Lin WY, Chang YC, Huang JL, et al. Relationship of myocardial substrate characteristics as assessed by myocardial perfusion imaging and cardiac reverse remodeling levels after cardiac resynchronization therapy. Ann Nucl Med 2016;30:484-93.

13. Chen J, Garcia EV, Folks RD, Cooke CD, Faber TL, Tauxe EL, Iskandrian AE. Onset of left ventricular mechanical contraction as determined by phase analysis of ECG-gated myocardial perfusion SPECT imaging: development of a diagnostic tool for assessment of cardiac mechanical dyssynchrony. J Nucl Cardiol 2005;12:68795.

14. AlJaroudi W. Early post-stress LV dyssynchrony: a new marker for significant CAD. J Nucl Cardiol 2014;21:1057-61.

15. Aljaroudi WA, Hage FG, Hermann D, Doppalapudi H, Venkataraman R, Heo J, et al. Relation of left-ventricular dyssynchrony by phase analysis of gated SPECT images and cardiovascular events in patients with implantable cardiac defibrillators. J Nucl Cardiol 2010;17:398-404.

16. Hage FG, Aggarwal H, Patel K, Chen J, Jacobson AF, Heo J, et al. The relationship of left ventricular mechanical dyssynchrony and cardiac sympathetic denervation to potential sudde $\mathrm{n}$ cardiac death events in systolic heart failure. J Nucl Cardiol 2014;21:78-85.

17. Chiang KF, Hung GU, Tsai SC, Cheng CM, Chang YC, Lin WY, et al. Impact of cardiac reverse remodeling after cardiac resynchronization therapy assessed by myocardial perfusion imaging on ventricular arrhythmia. J Nucl Cardiol 2017;24:1282-8.

18. Dorbala S, Vangala D, Sampson U, Limaye A, Kwong R, Di Carli MF. Value of vasodilator left ventricular ejection fraction reserve in evaluating the magnitude of myocardium at risk and the extent of angiographic coronary artery disease: a $82 \mathrm{Rb}$ PET/CT study. J Nucl Med 2007;48:349-58.

19. Dorbala S, Hachamovitch R, Curillova Z. Incremental prognostic value of gated $\mathrm{Rb}-82$ positron emission tomography myocardial perfusion imaging over clinical variables and rest LVEF. JACC Cardiovasc Imaging 2009;2:846-54.

20. Hung GU, Lee KW, Chen CP, Yang KT, Lin WY. Worsening of left ventricular ejection fraction induced by dipyridamole on Tl201 gated myocardial perfusion imaging predicts significant coronary artery disease. J Nucl Cardiol 2006;13:225-32.

21. Shen TY, Chang MC, Hung GU, Kao CH, Hsu BL. Prognostic value of functional variables as assessed by gated Tl-201 myocardial perfusion SPECT for major adverse cardiac events in patients with coronary artery disease. Acta Cardiol Sin 2013;29:243-50.

22. Huang WS, Huang CH, Lee CL, Chen CP, Hung GU. Relation of early post-stress left ventricular dyssynchrony and the extent of angiographic coronary artery disease. J Nucl Cardiol 2014;21:1048-56.

Publisher's Note Springer Nature remains neutral with regard to jurisdictional claims in published maps and institutional affiliations. 